This item was submitted to Loughborough's Research Repository by the author.

Items in Figshare are protected by copyright, with all rights reserved, unless otherwise indicated.

\title{
How periodic driving stabilizes and destabilizes Anderson localization on random trees
}

PLEASE CITE THE PUBLISHED VERSION

https://doi.org/10.1103/PhysRevB.103.L100204

\section{PUBLISHER}

American Physical Society

VERSION

VoR (Version of Record)

\section{PUBLISHER STATEMENT}

This paper was published in the journal Physical Review B and is available at https://doi.org/10.1103/PhysRevB.103.L100204.

\section{LICENCE}

CC BY-NC-ND 4.0

\section{REPOSITORY RECORD}

Roy, Sthitadhi, Roderich Moessner, and Achilleas Lazarides. 2021. "How Periodic Driving Stabilizes and Destabilizes Anderson Localization on Random Trees". Loughborough University.

https://hdl.handle.net/2134/16965301.v1. 


\title{
How periodic driving stabilizes and destabilizes Anderson localization on random trees
}

\author{
Sthitadhi Roy $\odot,{ }^{1,2}$ Roderich Moessner, ${ }^{3}$ and Achilleas Lazarides ${ }^{4}$ \\ ${ }^{1}$ Rudolf Peierls Centre for Theoretical Physics, Clarendon Laboratory, Oxford University, Parks Road, Oxford OX1 3PU, United Kingdom \\ ${ }^{2}$ Physical and Theoretical Chemistry, Oxford University, South Parks Road, Oxford OX1 3QZ, United Kingdom \\ ${ }^{3}$ Max-Planck-Institut für Physik komplexer Systeme, Nöthnitzer Straße 38, 01187 Dresden, Germany \\ ${ }^{4}$ Interdisciplinary Centre for Mathematical Modelling and Department of Mathematical Sciences, Loughborough University, \\ Loughborough, Leicestershire LE11 3TU, United Kingdom
}

(Received 15 January 2021; accepted 16 March 2021; published 23 March 2021)

\begin{abstract}
Motivated by the link between Anderson localization on high-dimensional graphs and many-body localization, we study the effect of periodic driving on Anderson localization on random trees. The time dependence is eliminated in favor of an extra dimension, resulting in an extended graph wherein the disorder is correlated along the new dimension. The extra dimension increases the number of paths between any two sites and allows for interference between their amplitudes. We study the localization problem within the forward scattering approximation (FSA), which we adapt to this extended graph. At low frequency, this favors delocalization as the availability of a large number of extra paths dominates. By contrast, at high frequency, it stabilizes localization compared to the static system. These lead to a regime of re-entrant localization in the phase diagram. Analyzing the statistics of path amplitudes within the FSA, we provide a detailed theoretical picture of the physical mechanisms governing the phase diagram.
\end{abstract}

DOI: 10.1103/PhysRevB.103.L100204

Localization in quantum systems [1-6] renders invalid the framework of statistical mechanics and paves the way to novel quantum phases and order in excited eigenstates [7]. Perhaps the most remarkable examples of these are the discrete time crystals in a periodically driven (Floquet) setting [8-11]. This spatiotemporal ordering is an inherently nonequilibrium phenomenon [12] apparently impossible in systems governed by static Hamiltonians [13]. While ergodic Floquet systems generically heat up to a featureless "infinite-temperature" state [14,15], breaking ergodicity robustly via disorder-induced localization prevents this heat death [16-19]. Understanding the mechanisms that might stabilize or destabilize localization in the presence of periodic driving is therefore a question of immanent interest.

While the problem of many-body localization (driven or otherwise) can also be equivalently viewed as one of singleparticle localization on the high-dimensional Fock-space graph, strong correlations in the latter render it qualitatively different from conventional Anderson localization on such graphs [20,21]. Nevertheless, a natural step towards a theoretical understanding of the mechanism governing localization in Floquet systems is to analytically study a simpler problem in a more controlled setting - the fate of Anderson transitions and localization on high-dimensional graphs. We, therefore, study Floquet tight-binding models defined on random highdimensional graphs which are generally treelike locally. In particular, the model we consider is described by a timeperiodic (with period $T \equiv 2 \pi / \omega$ ) Hamiltonian,

$$
H(t)=H_{0}+H_{1} \cos (\omega t),
$$

with $H_{0}=H_{\text {hop }}+H_{\text {dis }}$ and $H_{1}=H_{\text {hop }} . H_{\text {hop }}$ and $H_{\text {dis }}$ describe the hopping and the on-site disorder respectively,

$$
H_{\text {hop }}=\Gamma \sum_{\langle\alpha, \beta\rangle}|\alpha\rangle\langle\beta|+\text { H.c.; } H_{\text {dis }}=\sum_{\alpha} \epsilon_{\alpha}|\alpha\rangle\langle\alpha|,
$$

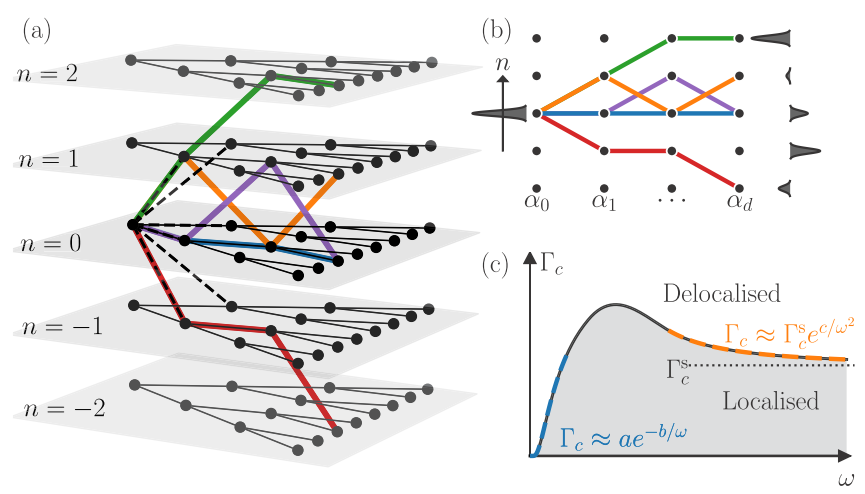

FIG. 1. (a) The Shirley picture for a driven tight-binding model on a tree. The different copies of the tree are indexed by $n$. The blue line shows the static path between the root site and another, say $\alpha_{0}$ and $\alpha_{d}$, whereas the different colored lines show some of the driving-induced paths between the root site and the same site's copy, $\left|\alpha_{d}, n\right\rangle$, on other Shirley layers. (b) Schematic of the FSA amplitude on sites $\left|\alpha_{d}, n\right\rangle$ starting from $\left|\alpha_{0}, 0\right\rangle$. The amplitudes on the former can be both positive and negative so they can interfere to give the total amplitude $\psi_{\alpha_{d}} \propto \sum_{n} \psi_{\alpha_{d}, n}$. (c) Summary of the localization phase diagram in the $(\Gamma, \omega)$ plane for the model in (a). At high frequencies, driving favors localiation and enhances the critical hopping compared to the static case, $\Gamma_{c}^{\mathrm{s}}$, whereas at low frequencies, it favors delocalization and $\Gamma_{c}$ decreases with decreasing $\omega$. 
respectively, where $\langle\alpha, \beta\rangle$ denotes links between pairs of sites on the graph, and the random potentials are drawn from a distribution with width $W$. Undriven cousins of the model in Eq. (1), i.e., disordered tight-binding models on trees and random regular graphs have long served as archetypes for localization transitions and related phenomena on highdimensional graphs, allowing for a remarkable amount of theoretical progress [22-37].

Following the approach pioneered by Shirley $[38,39]$, the periodic time dependence of a Hamiltonian of the form (1) can be eliminated in favor of an extra dimension. Generalizing the forward scattering approximation (FSA) [40] to this Shirley picture, we focus on the additional paths (compared to the static case) that the extra dimension allows for between any two physical sites on the graph. The interplay between these extra paths becoming available and their interference results in an amplification or attenuation of the amplitude relative to that of the static one. We provide analytical estimates for the locations of Anderson transitions in the hoppingstrength-frequency plane, Fig. 1. The phase diagram exhibits a characteristic re-entrant localised portion.

The remainder of this paper is organised as follows. We first recapitulate the Shirley formalism, showing how the time dependence is eliminated in favor of an extra dimension, then generalize the FSA to this situation. We then provide numerical evidence for the localization phase diagram [Fig. 1(c)], followed by analytical arguments elucidating the mechanisms underpinning it.

The problem in the Shirley picture maps to a new undriven system on an infinite ladder where each rung corresponds to a copy of the static part of the Hamiltonian $H_{0}$ with the $n^{\text {th }}$ rung shifted in energy by $n \omega$, and the driving Hamiltonian, $H_{1}$, mediating hopping between the rungs. Thus the new system lives on an extended graph with one additional dimension. This time-independent Hamiltonian, for the model described by Eqs. (1) and (2), takes the form

$$
\begin{aligned}
H_{F}= & \Gamma \sum_{\langle\alpha, \beta\rangle, n} \sum_{s=0, \pm 1}[|\alpha, n\rangle\langle\beta, n+s|+\text { H.c }] \\
& +\sum_{\alpha, n}\left(\epsilon_{\alpha}+n \omega\right)|\alpha, n\rangle\langle\alpha, n|
\end{aligned}
$$

where $|\alpha, n\rangle=|\alpha\rangle \otimes|n\rangle$ denotes a state localized on physical site $\alpha$ and Shirley rung $n$. Solutions of the timedependent Schrödinger equation $\left(i \partial_{t}-H(t)\right)|\phi(t)\rangle=0$ can be written as $\left|\phi_{a}(t)\right\rangle=e^{i \Omega_{a} t} \sum_{n}\left|\varphi_{a}^{(n)}\right\rangle e^{i n \omega t}$, where $\left|\varphi_{a}\right\rangle=$ $\sum_{n=-\infty}^{\infty}\left|\varphi_{a}^{(n)}\right\rangle \otimes|n\rangle$ is an eigenstate of the time-independent Hamiltonian (3) with eigenvalue $\Omega_{a}$ and $\left|\varphi_{a}^{(n)}\right\rangle$ is a state living in a single rung.

Physically, the hopping between different rungs corresponds to the system gaining or losing energy in integer multiples of $\omega$. Thus additional processes, or in the timeindependent picture, additional paths are made available to hop and delocalize away from a site $\alpha$ by the driving, see Fig. 1 for a visual representation. The question of localization for the driven system (1) is now mapped onto one for the time-independent system (3) with one extra dimension, which we will study within the FSA.

We begin by briefly describing the FSA for a static system. For a state initially (in the absence of hopping) localized at $\alpha_{0}$, the FSA estimates the amplitude on some other site, $\alpha_{d}$ at distance $d$, upon perturbatively including the effects of hopping by summing over all the shortest paths between the two sites. It is thus a stability analysis of the localized phase at $\Gamma=0$ for $\Gamma \neq 0$ [40]. The amplitude $\psi_{\alpha_{d}}$ is

$$
\psi_{\alpha_{d}}=\sum_{p: \alpha_{0} \rightarrow \alpha_{d}} \prod_{\beta \in p} \frac{\Gamma}{\epsilon_{\beta}-\epsilon_{\alpha_{0}}},
$$

where the sum is over all the shortest paths from $\alpha_{0}$ to $\alpha_{d}$. Breakdown of localization is indicated by the probability (over disorder realizations) of the state spreading to at least one site at arbitrarily large distance $d$ approaching unity. Defining $\left|\psi_{d}\right|^{2}=\max _{\alpha_{d}}\left|\psi_{\alpha_{d}}\right|^{2}$, the maximum amplitude over all sites at a given distance, the delocalization criterion is

$$
\lim _{d \rightarrow \infty} P\left(\frac{\ln \left|\psi_{d}\right|^{2}}{2 d}>-\xi^{-1}\right) \rightarrow 1,
$$

where $\xi$ is the localization length. Defining $\Lambda_{\alpha_{d}}=$ $\ln \left|\psi_{\alpha_{d}}\right|^{2} / 2 d-\ln (\Gamma / W)$ and $\Lambda_{d}=\max _{\alpha_{d}} \Lambda_{\alpha_{d}}$, the critical point $(\Gamma / W)_{c}$ can be expressed, using Eq. (5), as

$$
\begin{aligned}
& \lim _{d \rightarrow \infty} P\left(\Lambda_{d}>-\ln (\Gamma / W)_{c}\right) \rightarrow 1 \\
\Rightarrow & \lim _{d \rightarrow \infty} C\left(\Lambda_{d}=-\ln (\Gamma / W)_{c}\right) \rightarrow 1
\end{aligned}
$$

where $C\left(\Lambda_{d}\right)$ is the cumulative distribution and we have used the fact that the localization length $\xi$ diverges at the transition. In other words, if $P\left(\Lambda_{d}\right)$ is peaked at some value $\left\langle\Lambda_{d}\right\rangle$ then the critical point is at $\ln (\Gamma / W)_{c}=-\left\langle\Lambda_{d}\right\rangle$. Note that, as in the static case, taking the maximum amplitude over all sites at distance $d$ overestimates the critical disorder, resulting in an upper bound.

For the driven system at the $\Gamma=0$ limit, the $\left|\varphi_{a}\right\rangle$ are all localized on a single physical site and a single rung. Switching on $\Gamma$ allows for hopping to other sites and rungs. There is, however, one difference from the static case: for stroboscopic time evolution, $t=k T$ with integer $k,\left|\phi_{a}(k T)\right\rangle=$ $e^{i \Omega_{a} k T} \sum_{n}\left|\varphi_{a}^{(n)}\right\rangle$. Thus, the amplitude at a physical site $\alpha$ will depend on the amplitudes on all rungs, $\sum_{n}\left|\varphi_{a}^{(n)}\right\rangle$, implying that the probability amplitude of the state spreading to physical site $\alpha_{d}$ is $\psi_{\alpha_{d}}=\sum_{n} \psi_{\alpha_{d}, n}$ where $\psi_{\alpha_{d}, n}$ is the amplitude on site $\alpha_{d}$ and Shirley rung $n$.

To each path in the static system between two sites, say $\alpha_{0} \rightarrow \alpha_{1} \rightarrow \cdots \alpha_{d}$, there correspond $3^{d}$ paths in the driven system because at each hop in physical space the rung can change by 0 or \pm 1 due to our model being monochromatically driven. Thus, these paths all originate at $|\alpha, 0\rangle$ and terminate at $\left|\alpha_{d}, n_{d}\right\rangle$ with $n_{d} \in[-d, d]$ [41].

The total amplitude, $\psi_{\alpha_{d}}$ due to all these paths can then be written as

$$
\psi_{\alpha_{d}}=\sum_{p: \alpha_{0} \rightarrow \alpha_{d}} \sum_{q=1}^{3^{d}} \prod_{\alpha_{i} \in p} \frac{\Gamma}{\Delta_{i}+n_{\alpha_{i}}^{(q)} \omega},
$$

where $\Delta_{i}=\epsilon_{\alpha_{i}}-\epsilon_{\alpha_{0}}$, the first sum (over $p$ ) is over the physical paths between $\alpha_{0}$ and $\alpha_{d}$, the second sum (over $q$ ) is over the Shirley paths corresponding to the physical path $p, \alpha_{i}$ is the physical site on the $i^{\text {th }}$ step of the path, and $n_{i}^{(q)}$ is the rung index of the path $q$ at step $i$ [42]. The delocalization criterion for the driven system is then identical to that in Eq. (5) but 

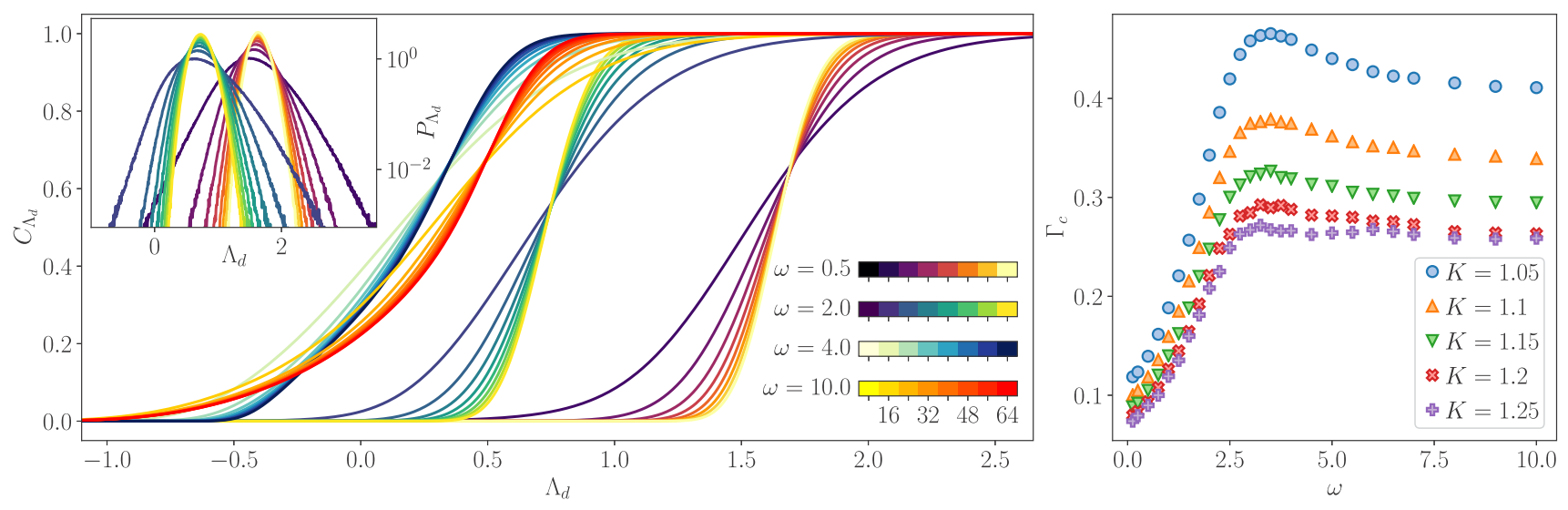

FIG. 2. Results for the localization phase diagram obtained from the FSA in the space of dimensionless parameters $\Gamma / W$ and $\omega / W$. We set $W=1$ and the disordered potential is drawn from a standard Normal distribution $\epsilon_{\alpha} \sim \mathcal{N}(0, W=1)$. The cumulative distribution of the rescaled FSA amplitudes, $C\left(\Lambda_{d}\right)$ [with $\left|\psi_{d}\right|^{2}$ obtained via Eq. (7)] for values of $d=8,16, \ldots, 64$ and four different values of $\omega$ labeled by the four color maps. The crossing points of the curves indicate the $\ln (1 / \Gamma)_{c}$ for the corresponding $\omega$. The inset shows the distributions $P\left(\Lambda_{d}\right)$ for $\omega=0.5$ and $\omega=2$ which get sharper with increasing $d$. Results are for $K=1.05$. (b) The critical $\Gamma_{c}$ as a function of $\omega$ for different values of $K$ obtained from the crossing of $C\left(\Lambda_{d}\right)$ exemplified in (a) and obtained using Eq. (8). Note the nonmonotonicity in $\Gamma_{c}$ as a function of $\omega$. Statistics for all data were obtained over $5 \times 10^{6}$ realizations.

with the FSA amplitude obtained in the Shirley picture from Eq. (7). In the notation used in Eq. (7), the path present in the static case is the one for which $n_{\alpha_{i}}^{(q)}=0$ for all $i$.

It is important to distinguish between the various interference effects at play here. For a given $n, \psi_{\alpha_{d}, n}$ receives contributions from multiple paths, each of which can be written as $\left|\alpha_{0}, n_{0}\right\rangle \rightarrow \cdots \rightarrow\left|\alpha_{j}, n_{j}\right\rangle \rightarrow \cdots \rightarrow\left|\alpha_{d}, n_{d}\right\rangle$ with $n_{0}=0$ and $n_{d}=n$. In particular, there are (i) different physical paths that follow the same Shirley rungs, so the $\alpha_{j}$ are different for each path for $j \neq 0, d$ but the $n_{j}$ the same for all; this is present for the static case too: (ii) the same physical path on different rungs, so the $\alpha_{j}$ are the same for all but the $n_{j}$ different. In addition, (iii) since $\psi_{\alpha_{d}}=\sum_{n} \psi_{\alpha_{d}, n}$, the FSA amplitudes $\psi_{\alpha_{d}, n}$ on different rungs interfere with each other. Crucially, neither (ii) or (iii) is present for the static case [43]. Equation (7) manifestly takes all of these kinds of interference into account.

In order to single out the effect of periodic-driving and interference between the Shirley paths, we take our physical graph to be a rooted tree, which has no loops. There then exists a unique shortest physical path between any two sites of the graph, removing the interference effect labeled (i) above and leaving only the driving-induced effects (ii) and (iii).

For a tree with average branching number $K$ [44], there are $K^{d}$ sites at distance $d$ from the root and one needs the probability distribution of the maximum amplitude over these $K^{d}$ sites, see above Eq. (5). As the amplitudes on each of these sites are independent and identically distributed with $P$, denoting this distribution by $P_{\max }\left(\Lambda_{d}\right)$, one finds

$$
P_{\max }\left(\Lambda_{d}\right)=K^{d}\left[C\left(\Lambda_{d}\right)\right]^{K^{d}-1} P\left(\Lambda_{d}\right),
$$

with $P, C$ the distribution and cumulative distribution for a single site. Equivalently, $C_{\max }\left(\Lambda_{d}\right)=\left[C\left(\Lambda_{d}\right)\right]^{K^{d}}$. It is thus possible to compute $P_{\max }\left(\Lambda_{d}\right)$ or $C_{\max }\left(\Lambda_{d}\right)$ for arbitrary $K$ by considering only single physical paths of length $d$. We, therefore, use the notation $\Lambda_{d}$ for both the amplitude of a single physical path and the maximum amplitude. The FSA for the former in the Shirley picture can be efficiently implemented using a transfer matrix [45].

In Fig. 2 we show the numerical results from the FSA in the Shirley picture. The inset in panel (a) shows that the distributions $P\left(\Lambda_{d}\right)$ sharpen with increasing $d$. This is manifested in the cumulative distributions, $C\left(\Lambda_{d}\right)$ for various $d$ crossing at a particular value of $\Lambda_{d}$ as evidenced in panel (a). The crossing point from Eq. (6) can be inferred to be $\ln (W / \Gamma)_{c}$, yielding the critical point. The critical $\Gamma_{c} / W$ so obtained is shown as a function of the frequency $\omega$ in panel (b). There are two crucial features of note: (i) For $\omega / W \gg 1, \Gamma_{c} / W$ is larger than that of the undriven system, $\Gamma_{c}^{\mathrm{s}} / W$ [46]. Thus, in this regime, the periodic driving enhances the localized phase. (ii) In the regime of $\omega / W \lesssim 1, \Gamma_{c} / W$ decreases with decreasing $\omega$ and is, in fact, much smaller than $\Gamma_{c}^{\mathrm{s}} / W$. Hence, in this regime, the driving suppresses the localized phase, favoring delocalization, and parametrically so with decreasing $\omega$.

In the following we present analytical arguments which give insight into the aforementioned behavior of $\Gamma_{c}$ with $\omega$. As the distributions for a system with arbitrary $K$ are simply related to that for a single physical path via Eq. (8), which leads to the qualitative behavior of $\Gamma_{c}$ with $\omega$ being the same for all $K$, it suffices to analyze the FSA amplitudes for different Shirley paths corresponding to a single physical path $\alpha_{0} \rightarrow \alpha_{1} \rightarrow \cdots \alpha_{d}$. It will be useful to write $\Lambda_{d}=\ln \left[\left(\sum_{q=1}^{3^{d}} w_{q}\right)^{2}\right] / 2 d$, where $(\Gamma / W)^{d} w_{q}$ is the amplitude of Shirley path $q$. Further, since the distribution of $\Lambda_{d}$ gets sharper with increasing $d$, one may take $\lim _{d \rightarrow \infty}\left\langle\Lambda_{d}\right\rangle=$ $-\ln (\Gamma / W)_{c}$. It will also be useful to decompose $\Lambda_{d}$ into direct and interference terms,

$$
\Lambda_{d}=\underbrace{\frac{1}{2 d} \ln \sum_{q} w_{q}^{2}}_{\Lambda_{d}^{\text {dir }}}+\underbrace{\frac{1}{2 d} \ln \left(1+\frac{\sum_{q \neq q^{\prime}} w_{q} w_{q^{\prime}}}{\sum_{q} w_{q}^{2}}\right)}_{\Lambda_{d}^{\text {int }}} .
$$

We first turn to the low-frequency regime, $\omega / W \ll 1$. We may drop the interference between the paths, approximating 


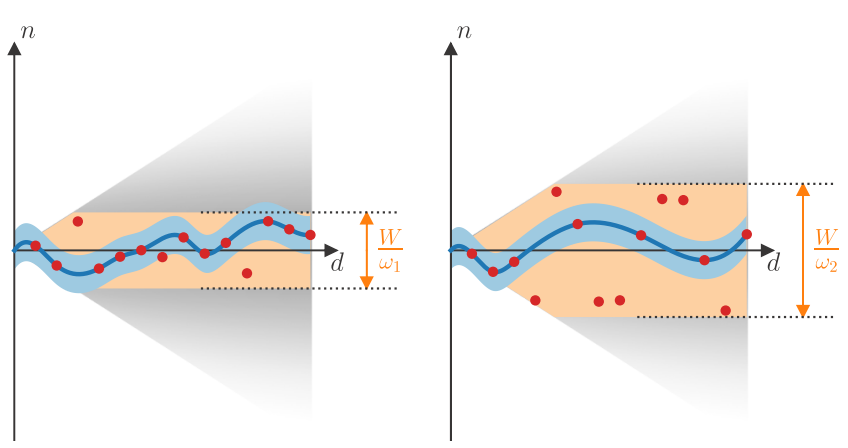

FIG. 3. Schematic picture for the Shirley resonances in the low-frequency regime, $\omega \ll W$. In the plots above $\omega_{1}>\omega_{2}$. The resonances denoted by the red dots, where the effective site energies $\left|\Delta_{i}+n_{\alpha_{j}}^{*} \omega\right|$ are within $\omega$ typically stay within a strip (shaded in orange) in the Shirley direction of width $W / \omega$. The larger $\omega / W$, the greater the likelihood of a resonance occurring per unit length. A path $q$ is resonant if the $n_{\alpha}^{(p)}$ passes within a distance 1 of these resonances, as indicated by the blue-shaded "swept area" of each path.

$\Lambda_{d} \approx \Lambda_{d}^{\text {dir }}$ in this regime. This is justified because [45], firstly, for $\omega / W \ll 1,\left|\Lambda_{d}^{\mathrm{int}}\right| \ll\left|\Lambda_{d}^{\mathrm{dir}}\right|$ and secondly $\Lambda_{d}^{\mathrm{int}}<0$ so that ignoring it overestimates $(W / \Gamma)_{c}$, consistent with the general bounds placed by the FSA.

For a given path $q,\left|n_{\alpha_{i}}^{(q)}-n_{\alpha_{i+1}}^{(q)}\right|=0$ or 1 , so a path selected at random from all possible paths is a simple random walk with $\alpha$ playing the role of time. On the other hand, the $\epsilon_{\alpha}$ are uncorrelated for different $\alpha$. We define a physical site $\alpha_{i}$ to be resonant on a Shirley rung $n_{\alpha_{i}}^{*}$ if $\left|\Delta_{i}-n_{\alpha_{i}}^{*} \omega\right|<\omega$ such that all the resonances lie within a strip of width $\propto W / \omega$ in the Shirley direction (see Fig. 3). Hence, for a typical path, the probability of a resonance vanishes after $\gg(W / \omega)^{2}$ steps since by then the random walk is well outside the strip of resonances. Although in the limit of $d \rightarrow \infty$, a fraction tending to 1 of these paths eventually do escape this strip eliminating the possibility of a resonance, driving can still cause delocalization as $\Lambda_{d}$ consists of a sum of of the contributions of the paths and not an average. One thus needs to consider an effective number of paths $N_{q}^{\text {eff }}$ which is the sum, over all the paths, of the fraction of each path spent inside the strip.

For simplicity, consider a single path lying entirely within the strip. As it meanders along the strip (Fig. 3) the probability it picks up a resonance per step is constant, $\rho(\omega)$, the same for all steps (due to the uncorrelated nature of the $\epsilon_{\alpha}$ ). A path of length $d$ will therefore pick up $\mu=\rho(\omega) d$ resonances on average. Over the ensemble of all paths lying entirely in the strip, the probability that a given path has $r$ resonances is Poisson, $P_{r}=\exp (-\mu) \mu^{r} / r$ !. Finally, for a given path $q$, $w_{q}=\prod_{i=1}^{d}\left(\Delta_{i}+n_{\alpha_{i}}^{(q)}\right)^{-1}$, we replace the factor for $\alpha_{i}$ with $1 / W$ if $\alpha_{i}$ is off-resonant, $1 / \omega$ if resonant; thus a path with $r$ resonances will have $w_{q} \approx(W / \omega)^{2 r}$. From all the above together, we have $\Lambda_{d} \approx \frac{1}{2 d} \ln \left(N_{q}^{\text {eff }} \sum_{r=0}^{d} P_{r} \frac{W^{2 r}}{\omega^{2 r}}\right)$. In the limit of $d \rightarrow \infty$,

$$
\lim _{d \rightarrow \infty} \Lambda_{d} \approx c-\frac{1}{2} \rho(\omega)\left(1-\frac{W^{2}}{\omega^{2}}\right),
$$

where $c \equiv \lim _{d \rightarrow \infty}\left(\ln N_{q}^{\text {eff }}\right) / 2 d$ is an $\mathcal{O}(1)$ constant [45]. As a site $\alpha_{i}$ on some path $q$ is resonant when $\left|\Delta_{i}+n_{\alpha_{i}}^{(q)}\right|$ is within $\omega$, a rough estimate for the probability of a resonance $\rho(\omega)$ is $\omega / W$. With this estimate, the localization critical point is

$$
(\Gamma / W)_{c} \approx a \exp (-b W / \omega),
$$

where $a$ and $b$ are constants. Thus, in this regime, driving destabilizes the localized phase (suppressing $\Gamma_{c}$ ) as one might expect $[16,17,19]$. Note that this is a result of the competition between the increase in the number of resonances (since $\rho(\omega)=\omega / W)$ and the reduction of their strength $(1 / \omega)$ as $\omega$ increases.

Next we turn to the high-frequency regime, $\omega / W \gg 1$, where the driving enhances localization as indicated by the increase in $\Gamma_{c}$ compared to $\Gamma_{c}^{\mathrm{s}}$. We first note that for $\omega=\infty$, only the static path contributes, the amplitude for which we denote as $w_{\mathrm{s}}$. We will therefore use it as a reference and consider the effect of deviations from it. For large $\omega / W \gg$ 1 , Shirley paths that deviate from the static path at $x$ sites have an amplitude relative to the static one suppressed parametrically as $\mathcal{O}\left((W / \omega)^{x}\right)$. The leading order contribution (at $\left.\mathcal{O}\left((W / \omega)^{2}\right)\right)$ can be shown to be [45]

$$
\Lambda_{d}(\omega) \approx \frac{1}{2 d} \ln \left[w_{\mathrm{s}}^{2}\left(1-\frac{4}{\omega^{2}} \sum_{i=1}^{d} \Delta_{i}^{2}+\frac{\Delta_{d-1} \Delta_{d}}{\omega^{2}}\right)\right] .
$$

Equation (12) already shows that $\Lambda_{d}$ in the presence of driving is smaller than that of the static case, $\Lambda_{d}^{\mathrm{s}}=\ln w_{\mathrm{s}}^{2} / 2 d$, for any disorder realization indicating an enhancement of the localized phase. Using the fact that the $\Delta_{i}$ 's are independent of each other and $\omega / \Delta_{i} \gg 1$, Eq. (12) gives

$$
\left\langle\Lambda_{d}(\omega)\right\rangle \stackrel{d \gg 1}{\approx}\left\langle\Lambda_{d}^{\mathrm{s}}\right\rangle-4 W^{2} / \omega^{2} .
$$

Since the distribution of $\Lambda_{d}$ sharpens with increasing $d$ [see Fig. 2(a)], it seems reasonable to assume that $\lim _{d \rightarrow \infty}\left\langle\Lambda_{d}\right\rangle=$ $-\ln (\Gamma / W)_{c}$, as such Eq. (13) yields,

$$
\Gamma_{c}(\omega) \approx \Gamma_{c}^{\mathrm{s}} e^{4 W^{2} / \omega^{2}} .
$$

The result in Eq. (14) explicitly shows the increase in $\Gamma_{c}$ from $\Gamma_{c}^{\mathrm{s}}$ and the enhancement of the localized phase.

The mechanism behind the driving-induced suppression of the total FSA amplitude can be understood as follows. For every site $\alpha_{i}$ on the physical path, there exists a pair of Shirley paths with $n_{\alpha_{j}}^{(q)}= \pm \delta_{i j}$. If we consider, without loss generality, $\Delta_{i}>0$, then $\Delta_{i} \pm \omega \gtrless 0$ with $\left|\Delta_{i}+\omega\right|>\left|\Delta_{i}-\omega\right|$. Hence, the path whose amplitude has the opposite (same) sign to the static path has a higher (lower) magnitude. The total amplitude is thus suppressed compared to the static case. However, the correction turns out to be $\mathcal{O}\left(1 / \omega^{2}\right)$ so that one has to take into account all paths which deviate at up to two sites from the static path.

The physical picture and the derivation of Eq. (12) suggest that this generalizes to pairs of paths that deviate at multiple sites from the static path, but with opposite signs of the Shirley rung indices. While the quantitative result in Eq. (14) can change upon including higher-order (in $1 / \omega$ ) contributions, localization enhancement is expected to stay robust; however, a concrete analytical demonstration remains for future work.

In summary, we have addressed the problem of Anderson localization on driven, disordered trees using the FSA on an extended Shirley graph by eliminating the time dependence of 
the Hamiltonian in favor of an extra dimension. The interplay between the availability of additional paths due to the driving and their interference yields a nontrivial localization phase diagram; in the high-frequency regime, the localized phase is stabilized relative to the undriven case, so that there is localization for weaker disorder than in the static case, whereas in the low-frequency regime driving destabilizes localization and increases the strength of the disorder required for localization.

A natural next step towards addressing the stability towards driving of many-body localization due to either correlations in Fock-space disorder [20] or constraints in the Fock-space graph [47] would be to incorporate the effect of multiple static paths between any two sites, rather than focus on purely treelike structures as we have done here. It is also conceivable that the framework discussed here can be used to analyze the problem of localization or lack thereof in quasiperiodically driven systems where one would require multiple Shirley directions, one for each of the frequencies present in the drive.

\section{ACKNOWLEDGMENTS}

S.R. thanks D. E. Logan, and R.M. thanks A. Scardicchio and S. Sondhi for discussions of the FSA. This work was in part supported by EPSRC Grant No. EP/S020527/1 and by the Deutsche Forschungsgemeinschaft under cluster of excellence ct.qmat (EXC 2147, Project id 390858490).
[1] P. W. Anderson, Absence of diffusion in certain random lattices, Phys. Rev. 109, 1492 (1958).

[2] D. M. Basko, I. L. Aleiner, and B. L. Altshuler, Metalinsulator transition in a weakly interacting many-electron system with localized single-particle states, Ann. Phys. 321, 1126 (2006)

[3] I. V. Gornyi, A. D. Mirlin, and D. G. Polyakov, Interacting Electrons in Disordered Wires: Anderson Localization and Low- $T$ transport, Phys. Rev. Lett. 95, 206603 (2005).

[4] V. Oganesyan and D. A. Huse, Localization of interacting fermions at high temperature, Phys. Rev. B 75, 155111 (2007).

[5] M. Žnidarič, T. Prosen, and P. Prelovšek, Many-body localization in the Heisenberg XXZ magnet in a random field, Phys. Rev. B 77, 064426 (2008).

[6] R. Nandkishore and D. A. Huse, Many-body localization and thermalization in quantum statistical mechanics, Annu. Rev. Condens. Matter Phys. 6, 15 (2015).

[7] D. A. Huse, R. Nandkishore, V. Oganesyan, A. Pal, and S. L. Sondhi, Localization-protected quantum order, Phys. Rev. B 88, 014206 (2013).

[8] V. Khemani, A. Lazarides, R. Moessner, and S. L. Sondhi, Phase Structure of Driven Quantum Systems, Phys. Rev. Lett. 116, 250401 (2016).

[9] D. V. Else, B. Bauer, and C. Nayak, Floquet Time Crystals, Phys. Rev. Lett. 117, 090402 (2016).

[10] N. Y. Yao, A. C. Potter, I.-D. Potirniche, and A. Vishwanath, Discrete Time Crystals: Rigidity, Criticality, and Realizations, Phys. Rev. Lett. 118, 030401 (2017).

[11] R. Moessner and S. L. Sondhi, Equilibration and order in quantum Floquet matter, Nat. Phys. 13, 424 (2017).

[12] H. Watanabe and M. Oshikawa, Absence of Quantum Time Crystals, Phys. Rev. Lett. 114, 251603 (2015).

[13] V. Khemani, R. Moessner, and S. L. Sondhi, A Brief History of Time Crystals, arXiv:1910.10745 [cond-mat.str-el].

[14] A. Lazarides, A. Das, and R. Moessner, Equilibrium states of generic quantum systems subject to periodic driving, Phys. Rev. E 90, 012110 (2014).

[15] L. D'Alessio and M. Rigol, Long-Time Behavior of Isolated Periodically Driven Interacting Lattice Systems, Phys. Rev. X 4, 041048 (2014).

[16] P. Ponte, Z. Papić, F. Huveneers, and D. A. Abanin, Many-Body Localization in Periodically Driven Systems, Phys. Rev. Lett. 114, 140401 (2015).
[17] A. Lazarides, A. Das, and R. Moessner, Fate of Many-Body Localization Under Periodic Driving, Phys. Rev. Lett. 115, 030402 (2015).

[18] P. Ponte, A. Chandran, Z. Papić, and D. A. Abanin, Periodically driven ergodic and many-body localized quantum systems, Ann. Phys. 353, 196 (2015).

[19] D. A. Abanin, W. De Roeck, and F. Huveneers, Theory of many-body localization in periodically driven systems, Ann. Phys. 372, 1 (2016).

[20] S. Roy and D. E. Logan, Fock-space correlations and the origins of many-body localization, Phys. Rev. B 101, 134202 (2020).

[21] S. Roy and D. E. Logan, Localization on Certain Graphs with Strongly Correlated Disorder, Phys. Rev. Lett. 125, 250402 (2020).

[22] R. Abou-Chacra, D. J. Thouless, and P. W. Anderson, A selfconsistent theory of localization, J. Phys. C 6, 1734 (1973).

[23] J. T. Chalker and S. Siak, Anderson localization on a Cayley tree: A new model with a simple solution, J. Phys.: Condens. Matter 2, 2671 (1990).

[24] F. Evers and A. D. Mirlin, Anderson transitions, Rev. Mod. Phys. 80, 1355 (2008).

[25] A. De Luca, B. L. Altshuler, V. E. Kravtsov, and A. Scardicchio, Anderson Localization on the Bethe Lattice: Nonergodicity of Extended States, Phys. Rev. Lett. 113, 046806 (2014).

[26] B. L. Altshuler, L. B. Ioffe, and V. E. Kravtsov, Multifractal states in self-consistent theory of localization: Analytical solution, arXiv:1610.00758 [cond-mat.dis-nn].

[27] K. S. Tikhonov, A. D. Mirlin, and M. A. Skvortsov, Anderson localization and ergodicity on random regular graphs, Phys. Rev. B 94, 220203(R) (2016).

[28] I. García-Mata, O. Giraud, B. Georgeot, J. Martin, R. Dubertrand, and G. Lemarié, Scaling Theory of the Anderson Transition in Random Graphs: Ergodicity and Universality, Phys. Rev. Lett. 118, 166801 (2017).

[29] M. Sonner, K. S. Tikhonov, and A. D. Mirlin, Multifractality of wave functions on a Cayley tree: From root to leaves, Phys. Rev. B 96, 214204 (2017).

[30] G. Biroli and M. Tarzia, Delocalization and ergodicity of the Anderson model on Bethe lattices, arXiv:1810.07545 [condmat.dis-nn].

[31] V. E. Kravtsov, B. L. Altshuler, and L. B. Ioffe, Non-ergodic delocalized phase in anderson model on bethe lattice and regular graph, Ann. Phys. 389, 148 (2018). 
[32] K. S. Tikhonov and A. D. Mirlin, Critical behavior at the localization transition on random regular graphs, Phys. Rev. B 99, 214202 (2019).

[33] S. Savitz, C. Peng, and G. Refael, Anderson localization on the Bethe lattice using cages and the Wegner flow, Phys. Rev. B 100, 094201 (2019).

[34] I. García-Mata, J. Martin, R. Dubertrand, O. Giraud, B. Georgeot, and G. Lemarié, Two critical localization lengths in the Anderson transition on random graphs, Phys. Rev. Research 2, 012020(R) (2020).

[35] M. Tarzia, Many-body localization transition in Hilbert space, Phys. Rev. B 102, 014208 (2020).

[36] G. Biroli and M. Tarzia, Delocalized glassy dynamics and many-body localization, Phys. Rev. B 96, 201114(R) (2017).

[37] G. Biroli and M. Tarzia, Anomalous dynamics on the ergodic side of the many-body localization transition and the glassy phase of directed polymers in random media, Phys. Rev. B 102, 064211 (2020).

[38] J. H. Shirley, Solution of the Schrödinger equation with a Hamiltonian periodic in time, Phys. Rev. 138, B979 (1965).

[39] H. Sambe, Steady states and quasienergies of a quantummechanical system in an oscillating field, Phys. Rev. A 7, 2203 (1973).

[40] F. Pietracaprina, V. Ros, and A. Scardicchio, Forward approximation as a mean-field approximation for the Anderson and many-body localization transitions, Phys. Rev. B 93, 054201 (2016).
[41] The Shirley Hamiltonian (3) is translation-invariant in the Shirley direction and the initial state's Shirley index can be chosen to be $n=0$ without loss of generality.

[42] Formally, $n_{i}^{(q)}=\sum_{i=1}^{q} s_{i}^{(q)}$ where $s_{i}^{(q)}= \pm 1,0$ is the change in the Shirley index on path $q$ at step $i$.

[43] The role of interferences of this kind were studied in a different context of transport in a driven one-dimensional Anderson insulator [48].

[44] $K$ can be fractional as well. For instance, if a fraction $p$ and $1-p$ of the sites have $K_{1}$ and $K_{2}$ descendants respectively, the average branching number is $K=p K_{1}+(1-p) K_{2}$.

[45] See Supplemental Material at http://link.aps.org/supplemental/ 10.1103/PhysRevB.103.L100204 for details on (i) transfer matrix for FSA in Shirley picture, (ii) FSA amplitudes at high-frequency, (iii) the statistics of Shirley path amplitudes, and (iv) effective number of paths in low-frequency regime.

[46] The undriven system is obtained by taking the $\omega \rightarrow \infty$ limit, which is equivalent to having one Shirley copy. $\Gamma_{c}^{\mathrm{s}}$ is obtained by computing the FSA amplitudes on this graph with a single Shirley copy.

[47] S. Roy and A. Lazarides, Strong ergodicity breaking due to local constraints in a quantum system, Phys. Rev. Research 2 , 023159 (2020).

[48] K. Agarwal, S. Ganeshan, and R. N. Bhatt, Localization and transport in a strongly driven Anderson insulator, Phys. Rev. B 96, 014201 (2017). 